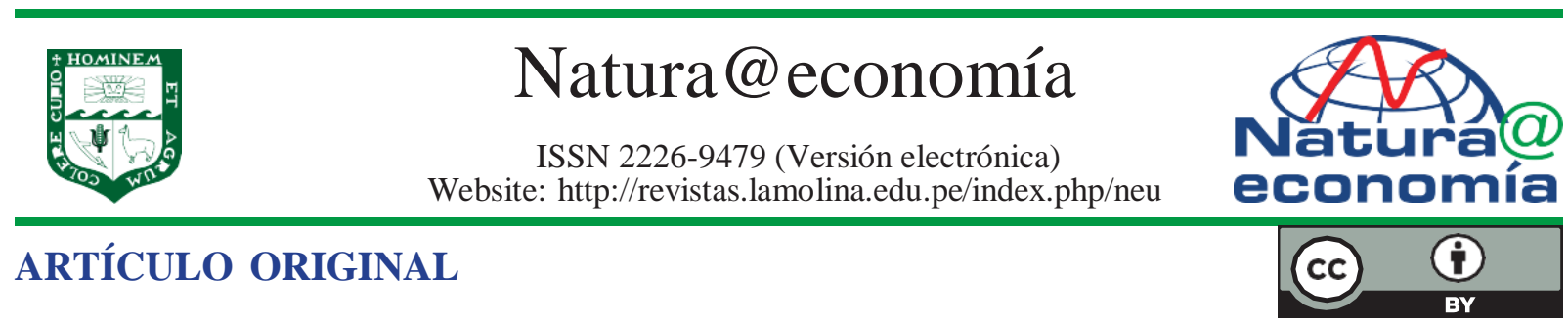

\title{
Costo económico de la mortalidad por cáncer de pulmón asociado al material particulado en el área de Lima Metropolitana
}

\section{Economic Cost of Mortality from Lung Cancer Related to Particulate Matter in Lima Metropolitan Area}

\author{
Sabrina Estefanía Díaz ${ }^{*}$; Cindy Silva ${ }^{2}$; Raymundo Jesús Mogollón ${ }^{3}$; \\ Carlos Enrique Orihuela ${ }^{4}$
}

\begin{abstract}
${ }^{1}$ Universidad Nacional Agraria La Molina, Lima, Perú. ID de ORCID: 0000-0001-7976-1440, sdiaz@lamolina.edu.pe
${ }^{2}$ Universidad Nacional Agraria La Molina, Lima, Perú. ID de ORCID: 0000-0003-0133-7775, cindysilva@ lamolina.edu.pe

${ }^{3}$ Universidad Nacional Agraria La Molina, Lima, Perú. ID de ORCID: 0000-0003-2130-2051, raymundo.mogollon@ gmail.com

${ }^{4}$ Universidad Nacional Agraria La Molina, Lima, Perú. ID de ORCID: 0000-0002-5787-0950, corihuela@lamolina.edu.pe
\end{abstract}

Recepción: 22/3/2021; Aceptación: 29/4/2021

\section{Resumen}

El cáncer de pulmón es una enfermedad respiratoria que presenta el mayor número de diagnósticos durante los últimos años, siendo la causa más común de muerte por cáncer a nivel mundial. Además del tabaquismo, las altas concentraciones de material particulado han aumentado significativamente los registros de esta enfermedad principalmente en países en desarrollo con grandes poblaciones. En Lima Metropolitana (LM), el cáncer de pulmón corresponde a la segunda causa de muerte por cáncer y, si bien se han aplicado medidas regulatorias que intentan mitigar la contaminación del aire, poco se ha estudiado sobre el costo económico asociado a esta enfermedad. Por ello, este estudio contribuye a la literatura mediante la estimación del costo económico del cáncer de pulmón como consecuencia de las concentraciones de material particulado en LM, durante el periodo 2010-2019. Para ello, se utilizaron los factores de la función concentración-respuesta a fin de estimar el número de casos de mortalidad por cáncer de pulmón asociados a la concentración de material particulado. Los resultados indican que el costo fluctuó entre 0,037\% y 0,122 \% del Producto Bruto Interno de LM. A pesar de que este

Forma de referenciar el artículo: Díaz, S., Silva, C., Mogollón, R. y Orihuela, C. (2021). Costo económico de la mortalidad por cáncer de pulmón asociado al material particulado en el área de Lima Metropolitana. Natura@ economía, 6(1), 28-42. http://dx.doi.org/10.21704/ne.v6i1.1729

DOI: http://dx.doi.org/10.21704/ne.v6i1.1729

* Autor de correspondencia: Díaz, S. Email: sdiaz@lamolina.edu.pe

CLos autores. Publicado por la Universidad Nacional Agraria La Molina. 
porcentaje es relativamente bajo, se ha incrementado aproximadamente un $8,6 \%$ a lo largo del período y, por tanto, es importante atender este problema con la aplicación de políticas públicas adecuadas, tanto en el ámbito de salud como el ambiental.

Palabras clave: Material particulado; mortalidad; cáncer de pulmón; Lima Metropolitana.

\begin{abstract}
Lung cancer is a respiratory disease that shows a larger number of diagnostics during the last years, being the most common cause of death from cancer around the world. Also smoking, the highest concentration of particulate matter has increased significantly the registers of this disease, mainly in develop countries with large population. In Lima Metropolitan (LM), lung cancer corresponds to the second cause of cancer death, so the regulatory measures that have been applied, they intent to mintage the air pollution, the economic cost has been little studied related to this disease. Therefore, this study contributes to literature through the estimation of the economic cost from lung cancer like consequence of the concentrations of particulate matter in LM, during the period from 2010 to 2019. For this reason, the function factors of concentrateresponse are used to estimate the number of the mortality cases from lung cancer related to the concentration of the particulate matter, and cost is found that fluctuated between $0,037 \%$ and $0,122 \%$ of PBI from Lima. Despite of this percentage is relatively low, it is increased approximately in $8,6 \%$ over the period and, therefore, it is important to attend this problem with the application of appropriate public policies, both in the field of health and the environment.

Keywords: Particulate matter; Mortality; Lung Cancer; Lima Metropolitan.
\end{abstract}

\section{Introducción}

Durante las últimas décadas, la contaminación del aire ha representado un grave problema para las ciudades de países en desarrollo. El acelerado proceso de industrialización y urbanización han provocado la intensificación de emisiones y concentraciones de contaminantes atmosféricos (Mannuci y Franchini, 2017; Fotourehchi, 2016; WHO, 2006). La literatura epidemiológica establece una relación entre la contaminación del aire y la salud humana, lo cual se manifiesta a través de una variedad de enfermedades (Landrigan et al., 2018; Cohen et al., 2017; Wang et al., 2016; Evans et al., 2013; WHO, 2006). Esto sugiere que una gran parte de la población que vive en países en desarrollo está potencialmente expuesta a complicaciones en la salud por este motivo.

Lima Metropolitana (LM) es una de las treinta grandes ciudades a nivel mundial con las concentraciones más altas de contaminantes del aire. Más del 75\% del año, presenta significativos niveles de concentración de diversos contaminantes a los que aproximadamente el $80 \%$ de la población está expuesta (WHO, 2020; Bell et al., 2011). El MP está constituido por partículas con un diámetro menor a 10 micras (conocidos como PM10) y por partículas con un diámetro menor a 2.5 micras (conocidos como PM2.5). El PM10 es originado por la re-suspensión del polvo de las carreteras, las cuales son generadas por el viento o vehículos en movimiento, mientras que las partículas finas (PM2.5) se derivan directamente del proceso de combustión vehicular, industria y generación de energía (Vu et al., 2019; Tapia et al., 2018; Mannucci y Franchini, 2017; Krzyżanowski et al., 2005).

Con respecto al inventario de emisiones en LM (año base 2016), y en relación con el PM2.5, la fuente que aporta mayores concentraciones de material particulado corresponde al conjunto de fuentes móviles con un $58 \%$, seguido de las fuentes fijas 
puntuales $(26 \%)$ y las fuentes de área (16\%) (MINAM, 2019). Así también, usando modelos receptores para la contaminación del aire en LM, se determinó que una de las principales fuentes de contaminación por MP es la actividad vehicular (Ilizarbe et al., 2020). De todos los contaminantes, el MP es el único que supera los umbrales de concentración, incluso en zonas donde se asienta la mayor parte de la población de LM (Pacsi, 2016; MINSA, 2012). Esto es alarmante, ya que el daño causado por el MP en la salud humana es más severo que cualquier otro contaminante (Landrigan et al., 2018; Cohen et al., 2017; WHO, 2006) debido a que el MP puede alcanzar las porciones más profundas de las vías respiratorias, llegando incluso al torrente sanguíneo (Landrigan, et al., 2018; Mannucci y Franchini, 2017). De hecho, se ha encontrado relación entre las concentraciones de MP y diversos efectos en la salud como derrames cerebrales, enfermedades respiratorias y cáncer (PAHO, 2020). En este contexto, el cáncer de pulmón, así como también las enfermedades cardiopulmonares y cardiovasculares han sido asociadas con la contaminación del aire (WHO, 2004).

En 1988, un estudio de la Internacional Agency for Research on Cancer (IARC, 2014) clasificó las emisiones de los gases de escape de diésel como probablemente cancerígenos para los humanos. Esto fue enfatizado nuevamente en marzo de 2012 con un estudio del National Cancer Institute (NCI) sobre la exposición ocupacional a dichas emisiones en mineros subterráneos, que indicó un mayor riesgo de muerte por cáncer de pulmón en los trabajadores expuestos. Esta evidencia científica resultó suficiente para concluir que los gases de escape de diésel son una causa de cáncer de pulmón.

En 2008, el cáncer de pulmón representaba el mayor número de diagnósticos en todo el mundo (1,61 millones, $12,7 \%$ del total de casos) y la causa más común de muerte por cáncer en ambos sexos (1,38 millones, 18,2\% del total de muertos por cáncer). Las tasas de mortalidad más altas se encuentran en las mismas regiones donde las tasas de incidencia son altas, evidenciándose que la letalidad de esta enfermedad (Ferlay et al., 2010). Si bien hacia el 2020 el número de nuevos casos en el mundo subió a 2,2 millones (con 1,8 millones de muertes), cayó su porcentaje sobre el total de casos de cáncer registrados $(11,4 \%$ del total) (GLOBOCAN, 2020).

En América, para el año 2012, el cáncer de pulmón fue el tercer tipo de cáncer más común (más de 324000 nuevos casos) y la primera causa de muerte por cáncer (más de 262000 muertes) (PAHO, 2012). Según proyecciones de la PAHO, se espera que para el año 2030 existan más de 541000 nuevos casos y aproximadamente 445000 muertes por cáncer de pulmón en toda América (PAHO, 2012).

Para el caso de Perú, específicamente en LM, durante el período 2004-2005, el cáncer pulmón fue el quinto tipo de cáncer más frecuente, con 837 casos promedio por año (INEN, 2011). Este número se incrementó notablemente a 3121 en el período 20102012, con una tasa de incidencia estandarizada (TEE) de 11,2 casos por cada 100000 habitantes y situó a este tipo de cáncer como la segunda causa de muerte por cáncer en LM con un total de 2591 muertes (INEN, 2016). Estas estadísticas indican que la atención al cáncer de pulmón constituye un problema de salud pública por su elevada carga, en especial para los países en desarrollo, y por los costos asociados, los cuales se relacionan a la pérdida de productividad y a los costos del cuidado informal (Ramos et al., 2020).

Por todo lo expuesto, el objetivo de esta investigación es determinar el costo económico de la mortalidad por cáncer de pulmón asociado a la exposición a niveles elevados de concentración de PM10 y PM2.5 en Lima Metropolitana durante el periodo 2010-2019. El daño a la salud dependerá de la población expuesta y su crecimiento, dispersión de los contaminantes según las variables climáticas y/o geográficas, entre otros. Es importante construir una tendencia de ese costo que 
permita explicar la relación entre los niveles de concentración y la mortalidad por cáncer de pulmón y así, generar información útil para el diseño e implementación de políticas públicas.

Desde la perspectiva económica, es importante traducir el efecto del MP en el cáncer de pulmón en términos monetarios, ya que resulta útil para la toma de decisiones. Son escasos los estudios que han cuantificado monetariamente el efecto de la contaminación por PM10 en LM, tales como Sánchez (2017), Larsen y Strukova (2006) y Kroeger (2004). Estos artículos, al igual que muchos similares (Hadei et al., 2020; Yu et al., 2019; Wei y Shi, 2018; Quan y Shiqiu, 2015; Meisner et al., 2015; WB, 2013; Yaduma et al., 2013) solo consideran un año o un periodo muy corto a fin de tener una idea del costo económico que genera la presencia del material particulado en la salud humana. Esto puede generar una imagen distorsionada de su importancia social y, potencialmente, generar indicadores cuestionables en políticas públicas.

Para abordar este tema se estima la población afectada mediante el método tradicional de evaluación de impactos en la salud empleando funciones concentraciónrespuesta (CR), los niveles de concentración de MP y otras características poblacionales, las cuales son posteriormente traducidas $\mathrm{y}$ agregadas en términos monetarios mediante dos enfoques: bienestar y capital humano. El primero se ha convertido en el enfoque estándar para valorar los riesgos de mortalidad derivados de la contaminación en los países de ingresos altos, mientras que el segundo es más apropiado para el análisis financiero y para medir los costos de la contaminación en los indicadores de cuentas nacionales (WB e IHME, 2016).

Este artículo se encuentra estructurado de la siguiente manera. En la sección 2 se describen los métodos utilizados para calcular los casos de cáncer de pulmón asociados al PM2.5 y PM10 y el costo económico asociado en Lima Metropolitana. En la sección 3 se presentan los resultados, mientras que en la sección 4 se establecen las conclusiones de esta investigación.

\section{Materiales y métodos}

El análisis en esta investigación cubre el periodo 2010-2019, para el cual fue posible adquirir la mayor cantidad de información que permitió realizar las estimaciones. El desarrollo de esta sección consiste en los siguientes pasos: (i) caracterización de la región de estudio haciendo énfasis en la relación entre los aspectos geográficos y las concentraciones de MP, (ii) estimación de los casos de cáncer de pulmón asociados al material particulado y (iii) cuantificación del costo económico de esta enfermedad.

\section{Caracterización de la región de estudio y las emisiones de PM}

Lima Metropolitana es la capital del Perú y en las últimas décadas, LM ha experimentado un rápido proceso de industrialización $\mathrm{y}$ urbanización, por lo que es considerada el centro de desarrollo socioeconómico más importante del país. El Producto Bruto Interno (PBI) de LM fue de US\$ 59000 millones en el año 2019, lo que representó un incremento de más de $46 \%$ respecto del año 2010 (INEI, 2020; WB, 2020). La población de LM creció $12 \%$ durante el periodo $2010-2019$, de 8,2 a 9,3 millones de habitantes (INEI, 2017a; 2017b).

Los fenómenos atmosféricos y aspectos geográficos explican la dispersión de las emisiones de MP. Los vientos locales de dirección sur-suroeste, generados por el Anticiclón del Pacifico Sur (APS), trasladan el MP provocando que se acumule en el este y noreste de la ciudad, las zonas con mayor altitud son donde se presentan las más altas concentraciones de MP. Por el contrario, la zona oeste registra las concentraciones más bajas (Silva et al., 2017). Los niveles máximos y mínimos de PM10 se observan durante el verano e invierno, respectivamente. Estas fluctuaciones estacionales de los contaminantes son explicadas por las condiciones meteorológicas, así como el 
proceso de inversión térmica (Silva et al., 2018; Silva et al., 2017; Jelić y Bencetić, 2010). En el caso del PM2.5 en LM, los mayores niveles de concentración se han observado durante el otoño-invierno, cuyas oscilaciones podrían depender del distrito en donde se haya registrado (Silva et al., 2017; Pacsi, 2016).

Con respecto a la tendencia temporal de la concentración de MP en LM, hay consenso en que ha sido decreciente hasta tiempos recientes, pero no hay consenso en que ha sido un efecto generalizado en todas las estaciones de monitoreo. El uso de data del periodo 2007-2014 en todas las estaciones de monitoreo, Pacsi (2016) señala que la variación multianual del PM10 y PM2,5 muestra una tendencia descendente moderada para el primero y leve para el segundo. A partir de la serie histórica de PM10, Espinoza (2018) encontró una disminución moderada en casi todas las estaciones de monitoreo para el periodo, aunque identificó un aumento progresivo a partir del año 2014. Por otro lado, en base a la tendencia de largo plazo del estimador Theil-Sen, Silva et al. (2017) encontraron evidencia de reducción en las concentraciones anuales de PM10, aunque solo en dos estaciones de monitoreo durante el periodo 2010-2015.

Estimación de los casos de cáncer de pulmón asociados al material particulado

En la literatura, la asociación de los casos de mortalidad por PM10 o PM2.5 se realiza con información del nivel de concentraciones del contaminante, no de sus emisiones. Para tal efecto, es costumbre utilizar la función concentración - respuesta expuesta en la ecuación (1).

$$
\Delta \mathrm{K}=\mathrm{Pob}^{*} \mathrm{~T} *(1-1 / \mathrm{RR})
$$

Esta función es de uso frecuente en la literatura (Liao et al., 2020; Liangliang et al., 2020; Hadei et al., 2020; Yu et al., 2019; Han et al., 2019; Wei y Shi, 2018; Yaduma et al., 2013), donde " $\Delta K$ " es el número de casos de cáncer de pulmón, Pob es la población expuesta a niveles que superan determinado umbral del MP, T es la tasa de mortalidad por cáncer de pulmón y el término ("1-" “ 1 ” $\rho$ 'RR”) corresponde a la fracción atribuible de la población por exposición al MP, siendo RR el factor de riesgo relativo del cáncer pulmonar, el cual se obtiene de la función concentraciónrespuesta (CR).

En general, la función $\mathrm{CR}$ relaciona el aumento en los niveles de concentración de un contaminante ambiental con el aumento de las probabilidades de desarrollar diversos efectos en la salud. Esta relación adopta diversas formas funcionales (Peng y Dominici, 2008). En esta investigación se utiliza la forma básica log-lineal, especificada en la expresión (2), la cual es utilizada por su sencillez.

$$
\mathrm{RR}=\exp \left[\beta\left(P M^{f}-P M^{0}\right)\right]
$$

El parámetro " $\beta$ ” es el coeficiente de la función CR y caracteriza la forma y la magnitud de la curva $\mathrm{CR}, \mathrm{PM}^{f}$ es el nivel concentración promedio actual del contaminante, siendo $\mathrm{PM}^{0}$ su umbral medido en microgramos por metro cúbico $\left(\mu \mathrm{g} / \mathrm{m}^{3}\right)$.

Cabe mencionar que los casos de cáncer de pulmón por exposición pueden ocurrir en un corto o largo plazo y que la población afectada puede presentar enfermedades preexistentes las cuales estarían siendo exacerbadas por el MP. En esta investigación se asume que los efectos finales del cáncer de pulmón ocurren en un mismo año y que la población expuesta no presenta enfermedades precedentes, ni que se origina en factores hereditarios.

\section{Selección de los factores CR por tipo de contaminante}

Serevisaron diversos estudios epidemiológicos que calcularon los factores $\mathrm{CR}$ factibles de ser aplicados a LM. Los factores de CR asociados al cáncer de pulmón fueron estimados mediante estudios de cohorte (exposición de largo plazo), en los que la asociación entre el MP y su efecto en la salud humana 
fue monitoreada durante un largo periodo, asegurando así la variación suficiente en los componentes que influyen en esta medición (condiciones atmosféricas y geográficas, poblacionales, dispersión y capacidad de transporte del contaminante, entre otros), lo cual permite mejores resultados (Peng y Dominici, 2008).

En la Tabla 1 se muestran las funciones CR seleccionadas para el análisis para PM2.5 y PM10.

Tabla 1. Funciones de concentración-respuesta para el cáncer de pulmón, según tipo de contaminante.

\begin{tabular}{|c|c|c|c|c|}
\hline \multirow{2}{*}{ Contaminante } & \multicolumn{2}{|c|}{ Factores CR (Media y 95\% IC) } & \multirow{2}{*}{ Fuente } & \multirow{2}{*}{ Tipo de estudio } \\
\hline & RR (\%) & $\widehat{\beta}(\%)$ & & \\
\hline $\mathbf{P M}_{2.5}$ & $\begin{array}{c}1,05(1,02-1,08) \\
\left(5,8 \mu \mathrm{g} / \mathrm{m}^{3}\right)\end{array}$ & $\begin{array}{c}0,0084(0,0034-0,0133) \\
\left(1 \mu \mathrm{g} / \mathrm{m}^{3}\right)\end{array}$ & Evans et al. (2013) & Cohorte \\
\hline $\mathbf{P M}_{10}$ & $\begin{array}{c}1,08(1,04-1,13) \\
\left(10 \mu \mathrm{g} / \mathrm{m}^{3}\right)\end{array}$ & $\begin{array}{c}0,0077(0,0039-0,0122) \\
\left(1 \mu \mathrm{g} / \mathrm{m}^{3}\right)\end{array}$ & $\begin{array}{l}\text { Chen y Hoek } \\
\text { (2020) }\end{array}$ & Cohorte \\
\hline
\end{tabular}

Fuente: elaboración propia

Dado que no se encontraron los coeficientes $\beta$ necesarios para evaluar el cambio inicial en los niveles de concentración del PM, éstos fueron estimados mediante la información disponible de los RR. En el caso del $\mathrm{PM}_{2.5}$, el valor hallado en Evans et al. (2013) corresponde a un cambio en la concentración de $5,8 \mu \mathrm{g} / \mathrm{m}^{3}$, mientras que para el PM10 obtenido de Chen y Hoek (2020) corresponde a un cambio de 10 $\mu \mathrm{g} / \mathrm{m}^{3}$. Por lo tanto, a partir de la función CR en (2) se obtuvieron las estimaciones del $\hat{\beta}^{j}$ con un cambio en la cantidad correspondiente de $\mu \mathrm{g} / \mathrm{m}^{3}$.

$$
\hat{\beta}^{\mathrm{j}}=\frac{\ln (R R)}{\Delta \mu g / m^{3}}
$$

\section{Concentraciones de MP y población expuesta en Lima Metropolitana}

En LM, las concentraciones de MP son registradas en las estaciones de monitoreo de la Calidad del Aire de Servicio Nacional de Meteorología e Hidrología del Perú (SENAMHI) y el Ministerio de Salud (MINSA). Estas estaciones se localizan de forma asimétrica en 16 de los 43 distritos de LM.

Para estimar la población expuesta a las concentraciones de $\mathrm{PM}_{2.5}$ en LM, se utilizó el estudio de Vu et al. (2019), donde mediante técnicas de teledetección satelital se elaboraron mapas de distribución espacial anual de la concentración de $\mathrm{PM}_{2.5}$, según intervalo, para el periodo 2010-2016. Esta información fue superpuesta con mapas distritales construidos por los autores a fin de asignar la población expuesta según rango de concentraciones por año. Se utilizó el promedio del intervalo, considerándose solo a aquellos que excedieron el umbral de concentración de $25 \mu \mathrm{g} / \mathrm{m}^{3}$ (MINAM, 2017). Asimismo, se corroboró la presencia de espacios no habitados en algunos distritos con la ayuda de la herramienta Google Earth. Para el periodo 2017-2019 se asumió una distribución espacial similar al año 2016.

Respecto al $\mathrm{PM}_{10}$, solo estuvo disponible un mapa de distribución de la concentración según temporada (verano e invierno) del año 
2011, el cual fue elaborado por el MINSA (2012). Ante ello, una opción fue utilizar alguna de las ratios de concentraciones de $\mathrm{PM}_{2.5} / \mathrm{PM}_{10}$ en LM disponibles en la literatura (Pacsi, 2016; Silva et al., 2017; Vu et al., 2019) a fin de inferir la distribución de concentraciones anuales de $\mathrm{PM}_{10}$. Sin embargo, emplear esta ratio equivale a asumir que los mapas de concentraciones anuales del $\mathrm{PM}_{10}$ y $\mathrm{PM}_{2.5}$ son iguales.

Una alternativa fue utilizar el mapa del MINSA (2012) y asumir la distribución de la concentración de $\mathrm{PM}_{10}$ como constante para todo el periodo en estudio. Así, la población expuesta del año 2011 fue la suma ponderada de la población identificada en el verano (asumido en 3 meses) e invierno ( 9 meses), es decir, una proporción de 3/12 para verano y de $9 / 12$ para invierno en todo el periodo. El resto del proceso fue similar al caso del $\mathrm{PM}_{2.5}$, pero considerando un umbral de $50 \mu \mathrm{g} / \mathrm{m}^{3}$. Dado que los intervalos de concentración del mapa del $\mathrm{PM}_{10}$ tuvieron márgenes considerablemente más amplios en comparación al caso del $\mathrm{PM}_{2.5}$, por lo que se esperarían amplios resultados en la población expuesta para el caso del $\mathrm{PM}_{10}$.

La información de población a nivel distrital solo estuvo disponible para el periodo 2010-2015 (INEI, 2017a). El periodo 20162019 fue proyectado en base a la tasa de crecimiento poblacional distrital para Lima Metropolitana (INEI, 2017b).

\section{Tasa de mortalidad}

Dado que no se encontró la tasa de mortalidad por cáncer de pulmón en LM para cada año del periodo considerado, se utilizó la información disponible en el Registro de Cáncer de Lima Metropolitana, entre 2010 y 2012, que afirma que esta tasa fue de 9,22 por cada 100000 habitantes (INEN, 2016). Es importante destacar que los resultados podrían estar subestimados o sobreestimados, según haya sido la evolución de la enfermedad durante el periodo.
Costo económico de los impactos en la salud Esta investigación supone que el costo económico del cáncer de pulmón equivale al costo de mortalidad, a pesar de que algunos estudios han estimado el costo de morbilidad asociado a esta enfermedad (Arik et al., 2020). Este costo de mortalidad suele ser estimado mediante dos enfoques: el de bienestar (VSL) y el de capital humano (EFH).

El primero mide el costo asociado al incremento del riesgo de contraer una enfermedad mortal. Esto equivale a la tasa marginal de sustitución entre el riesgo de mortalidad y el ingreso (Cameron, 2010), que es calculado con metodologías de preferencias declaradas o reveladas (OECD, 2014). Este enfoque es más adecuado para los análisis de bienestar económico y es el más utilizado para valorar los riesgos de mortalidad asociados con la contaminación en países de altos ingresos. No hay estimaciones locales para VSL, pero el estudio de Mardones y Riquelme (2018) es el más reciente (aplicado a Chile y otros países de América Latina) que ha calculado el VSL para Perú (US\$ 0,48 millones para el año 2013). Esta cifra se utilizó para todo el período y proporciona un límite superior de los daños a la salud.

El segundo asume que cuando un individuo fallece prematuramente, sus futuros ingresos son truncados, ocasionando un costo social. Así, prevenir esa muerte significaría recuperar un ahorro positivo para impulsar el crecimiento económico (WB y IHME, 2016). Este enfoque es más apropiado para el análisis financiero y la medición de costos de la contaminación en los indicadores de las cuentas nacionales (WB y IHME, 2016). Para el caso peruano, solo se dispone información de Seminario (2017), quien mediante el enfoque de capital humano (EFH) para LM obtuvo el costo social por fallecimiento prematuro, estimado en $\mathrm{S} / 465,784$ para el año 2016. Dada la ausencia de más información, se consideró la cifra de Seminario para todo el periodo. Vale resaltar que el EFH utiliza información macroeconómica del ingreso 
nacional como punto de partida.

Las cifras monetarias referidas al EFH fueron convertidas a US\$ mediante el tipo de cambio promedio de 3,37 (S/US\$) del año 2016 reportado por el Banco Central de Reserva del Perú, mientras que el VSL de Mardones fue actualizado utilizando el tipo de cambio promedio de 2,70 (S/US\$) del año 2013 (BCRP, 2020).

\section{Resultados y discusión}

Si bien el PM2.5 es más agresivo a la salud humana en comparación al PM10 (Landrigan, et al., 2018; Mannucci y Franchini, 2017), la concentración en exceso de este último ha sido la responsable de la mayor cantidad de casos de mortalidad en LM (Tabla 2). De acuerdo a los resultados obtenidos, en promedio, hubo más casos de mortalidad por cáncer de pulmón asociados al PM10 (156 muertes) que al PM2.5 (34 muertes). Asimismo, el segundo se incrementó en un porcentaje mayor $(20,3 \%)$ a lo largo del periodo.

Tabla 2. Estimación de los casos de mortalidad por cáncer de pulmón en LM asociados a la concentración de MP para el periodo 2010-2019.

\begin{tabular}{cccc}
\hline Año & $\mathbf{P M}_{\mathbf{2 , 5}}$ & $\mathbf{P M}_{\mathbf{1 0}}$ & Total \\
\hline 2010 & 33 & 146 & 179 \\
\hline 2011 & 32 & 149 & 180 \\
\hline 2012 & 29 & 151 & 180 \\
\hline 2013 & 29 & 153 & 182 \\
\hline 2014 & 37 & 156 & 193 \\
\hline 2015 & 29 & 158 & 188 \\
\hline 2016 & 38 & 160 & 199 \\
\hline 2017 & 39 & 162 & 201 \\
\hline 2018 & 39 & 164 & 203 \\
\hline 2019 & 40 & 164 & 204 \\
\hline Promedio & 34 & 156 & 191 \\
\hline Tasa de Variación (en \%) & 20,3 & 12,4 & 13,8 \\
\hline
\end{tabular}

Fuente: elaboración propia 
Con respecto a los costos asociados, se trabajó bajo tres posibles escenarios (alto, medio y bajo) según el coeficiente de la función $\mathrm{CR}$ (beta) utilizado.

En la Tabla 3 se presentan los resultados para los costos estimados con el enfoque de capital humano y de bienestar. Considerando el escenario medio, el costo económico de la mortalidad por cáncer de pulmón asociado a las concentraciones de $\mathrm{PM}_{2.5}$ osciló entre los 4,8 y los 15,8 millones de dólares, en promedio, a lo largo del periodo, presentando una brecha menor a la del $\mathrm{PM}_{10}$, cuyo rango medio de variación del costo estuvo entre 21,6 y 71,6 millones de dólares. A pesar de esto, se determinó que entre el 2010 y el 2019, los costos asociados al $\mathrm{PM}_{2.5}$ variaron en una magnitud mayor que los costos correspondientes al $\mathrm{PM}_{10}(20,3 \%$ y $12,4 \%$, respectivamente).

Finalmente, para el mismo escenario, las estimaciones indican que las pérdidas económicas por cáncer de pulmón por MP, como porcentaje del PBI de LM, fueron relativamente bajas, arrojando una brecha entre el $0,037 \%$ y el $0,122 \%$. Asimismo, estos porcentajes aumentaron un $8,6 \%$ entre el primero y el último año del periodo considerado.

En varios países desarrollados del mundo se ha estimado este costo con el fin de diseñar políticas de salud pública, pero no hay datos similares sobre el costo del cáncer de pulmón en países de bajos y medianos ingresos (Ramos et al., 2020). Así, por ejemplo, en 2009 estos costos fueron de aproximadamente 126 billones de euros en la Unión Europea (Luengo et al.,2013), mientras que los costos médicos directos asociados al cáncer de pulmón en los Estados Unidos para el año 2011 se estimaron en alrededor de 88,7 billones de dólares (AHRQ, 2012). 
Tabla 3. Costos de la mortalidad por cáncer de pulmón asociados al material particulado (Millones de dólares del 2016).

\begin{tabular}{|c|c|c|c|c|c|c|c|c|c|c|c|c|c|c|}
\hline & & & 2010 & 2011 & 2012 & 2013 & 2014 & 2015 & 2016 & 2017 & 2018 & 2019 & Promedio & Var. (\%) \\
\hline \multirow{6}{*}{$\mathbf{P M}_{2.5}$} & \multirow{3}{*}{ EFH } & Bajo & 1,9 & 1,8 & 1,7 & 1,7 & 2,2 & 1,7 & 2,2 & 2,3 & 2,3 & 2,3 & 2,0 & 20,49 \\
\hline & & Medio & 4,6 & 4,4 & 4,0 & 4,0 & 5,2 & 4,0 & 5,3 & 5,4 & 5,4 & 5,5 & 4,8 & 20,30 \\
\hline & & Alto & 7,0 & 6,7 & 6,0 & 6,1 & 7,9 & 6,2 & 8,1 & 8,2 & 8,3 & 8,4 & 7,3 & 20,13 \\
\hline & \multirow{3}{*}{ VSL } & Bajo & 6,4 & 6,1 & 5,5 & 5,5 & 7,2 & 5,6 & 7,4 & 7,5 & 7,6 & 7,7 & 6,6 & 20,49 \\
\hline & & Medio & 15,1 & 14,5 & 13,1 & 13,2 & 17,1 & 13,4 & 17,6 & 17,8 & 18,0 & 18,2 & 15,8 & 20,30 \\
\hline & & Alto & 23,1 & 22,2 & 20,1 & 20,2 & 26,1 & 20,5 & 26,8 & 27,1 & 27,4 & 27,7 & 24,1 & 20,13 \\
\hline \multirow{6}{*}{$\mathbf{P M}_{10}$} & \multirow{3}{*}{ EFH } & Bajo & 11,5 & 11,7 & 11,9 & 12,1 & 12,3 & 12,5 & 12,6 & 12,8 & 12,9 & 13,0 & 12,3 & 12,56 \\
\hline & & Medio & 20,2 & 20,5 & 20,9 & 21,2 & 21,5 & 21,9 & 22,1 & 22,4 & 22,6 & 22,7 & 21,6 & 12,37 \\
\hline & & Alto & 28,4 & 28,8 & 29,3 & 29,8 & 30,2 & 30,7 & 31,1 & 31,4 & 31,7 & 31,8 & 30,3 & 12,17 \\
\hline & \multirow{3}{*}{ VSL } & Bajo & 38,2 & 38,8 & 39,4 & 40,1 & 40,7 & 41,4 & 41,9 & 42,3 & 42,8 & 43,0 & 40,9 & 12,56 \\
\hline & & Medio & 67,0 & 68,1 & 69,2 & 70,3 & 71,4 & 72,6 & 73,4 & 74,1 & 74,9 & 75,3 & 71,6 & 12,37 \\
\hline & & Alto & 94,1 & 95,6 & 97,1 & 98,7 & 100,3 & 101,9 & 103,0 & 104,1 & 105,2 & 105,6 & 100,5 & 12,17 \\
\hline \multirow{6}{*}{ Total } & \multirow{3}{*}{ EFH } & Bajo & 13,4 & 13,5 & 13,6 & 13,8 & 14,5 & 14,2 & 14,9 & 15,0 & 15,2 & 15,3 & 14,3 & 13,69 \\
\hline & & Medio & 24,8 & 24,9 & 24,8 & 25,2 & 26,7 & 25,9 & 27,4 & 27,7 & 28,0 & 28,2 & 26,4 & 13,83 \\
\hline & & Alto & 35,3 & 35,5 & 35,4 & 35,9 & 38,1 & 36,9 & 39,2 & 39,6 & 40,0 & 40,2 & 37,6 & 13,74 \\
\hline & \multirow{3}{*}{ VSL } & Bajo & 44,5 & 44,9 & 45,0 & 45,6 & 47,9 & 47,0 & 49,3 & 49,8 & 50,3 & 50,6 & 47,5 & 13,69 \\
\hline & & Medio & 82,1 & 82,6 & 82,3 & 83,5 & 88,5 & 86,0 & 91,0 & 92,0 & 92,9 & 93,4 & 87,4 & 13,83 \\
\hline & & Alto & 117,2 & 117,8 & 117,2 & 118,9 & 126,3 & 122,4 & 129,8 & 131,2 & 132,6 & 133,3 & 124,7 & 13,74 \\
\hline \multirow{6}{*}{$\begin{array}{c}\text { Porcentaje } \\
\text { del PBI } \\
(\%)\end{array}$} & \multirow{3}{*}{ EFH } & Bajo & 0,019 & 0,020 & 0,019 & 0,019 & 0,020 & 0,019 & 0,020 & 0,021 & 0,021 & 0,021 & 0,020 & 8,45 \\
\hline & & Medio & 0,035 & 0,037 & 0,036 & 0,035 & 0,037 & 0,035 & 0,037 & 0,038 & 0,039 & 0,039 & 0,037 & 8,59 \\
\hline & & Alto & 0,051 & 0,053 & 0,051 & 0,050 & 0,052 & 0,050 & 0,053 & 0,054 & 0,055 & 0,055 & 0,052 & 8,50 \\
\hline & \multirow{3}{*}{ VSL } & Bajo & 0,064 & 0,066 & 0,065 & 0,064 & 0,066 & 0,064 & 0,067 & 0,068 & 0,070 & 0,069 & 0,066 & 8,45 \\
\hline & & Medio & 0,118 & 0,122 & 0,118 & 0,117 & 0,122 & 0,116 & 0,123 & 0,126 & 0,129 & 0,128 & 0,122 & 8,59 \\
\hline & & Alto & 0,168 & 0,174 & 0,168 & 0,167 & 0,174 & 0,165 & 0,175 & 0,180 & 0,183 & 0,182 & 0,174 & 8,50 \\
\hline
\end{tabular}

Como se evidencia a nivel mundial, las muertes por cáncer de pulmón han crecido en los últimos años (GLOBOCAN, 2020), pero no se encontró información respecto al número de muertes por este tipo de cáncer en LM y, por ello, se utilizó la tasa estandarizada de 9,22 muertes cada 100.000 habitantes del período 2010 - 2012 (INEN,2016) para realizar las estimaciones de todo el período. Entonces, es de esperar que el número de muertes asociado a los altos niveles de concentración pueden estar subestimados para los años posteriores al 2012, ya que, probablemente, se presenten mayores tasas de mortalidad.
Por otro lado, aun cuando la tendencia temporal del costo económico es creciente (Tabla 3), ello puede no reflejar adecuadamente lo ocurrido durante el periodo de estudio. Puesto que el nivel de concentración anual del PM10 fue asumido como constante (debido a la falta de mapas de concentración anual para LM), de manera que la estimación de su costo económico dependió principalmente de la tasa de crecimiento de la población expuesta. Esto pudo significar una sobrestimación del costo, aunque la aplicación enfoque de capital humano debió reducir este exceso y eventualmente minimizar la distorsión. 
Para el caso del $\mathrm{PM}_{2.5}$, para el que sí se dispuso de mapas de concentración para el periodo 2010-2016, los niveles de concentración descienden hasta el 2013 y luego suben. La misma tendencia se puede observar en las estimaciones de los casos de mortalidad (Tabla 2) y los costos asociados (Tabla 3). Es decir, para el PM2.5 se aprecia una relación directa entre el nivel de concentración de material particulado y su costo económico.

Vale la pena distinguir la concentración de MP registrada en una estación de monitoreo y aquella concentración estimada según modelos de exposición, los cuales suelen generar mapas de concentraciones. Los registros de algunas estaciones de monitoreo en LM han presentado una tendencia a la baja durante los últimos años (Pacsi, 2016; Espinoza, 2018). Pero ello no ha sido reflejado en los resultados de modelos de exposición. Si un mapa de concentraciones de PM contiene un estrecho intervalo de 50-55 $\mu \mathrm{g} / \mathrm{m} 3$, no hay forma de evidenciar la disminución en el tiempo de esa concentración (por ejemplo, de 54 a $51 \mu \mathrm{g} / \mathrm{m} 3)$. Esta es una limitación de este tipo de mapas para estimar de forma más precisa la magnitud del efecto en la salud de la población expuesta.

\section{Conclusiones}

Durante el periodo 2010-2019, el costo de la mortalidad por cáncer de pulmón asociados a la contaminación osciló entre $0,037 \%$ y $0,122 \%$ del PBI de LM. Si bien estos porcentajes son sustancialmente bajos, resulta preocupante el incremento de $8,6 \%$ que ha experimentado a lo largo del periodo. Por esa razón que es importante atender este problema con la aplicación de políticas públicas adecuadas, tanto en el ámbito de salud como el ambiental. Es importante señalar que estos costos estimados generalmente están "ocultos", ya que normalmente no se cuantifican ni se comparan con el valor de la actividad económica que generó la contaminación (es decir, el PIB). Estos resultados de salud se pueden prevenir en gran medida mediante políticas coordinadas que podría reducir efectivamente la exposición de la población a los contaminantes del aire de partículas inhalables.

Por el lado de las políticas de salud, debe incrementarse el monto del presupuesto destinado a la prevención y control de este tipo de cáncer en LM. Para el año 2018, el presupuesto destinado a atender todos los tipos de cáncer en el Perú fue de S/ 733,239 millones (MINSA, 2018), equivalentes a US\$ 192,5 millones actualizado a moneda del 2016. Es decir, ese monto resulta bajo, de acuerdo con las estimaciones de este artículo, ya que el costo de la mortalidad por cáncer de pulmón en LM oscila entre el 13,7\% y el $40,7 \%$ del presupuesto nacional de ese año.

En paralelo, se sugiere incrementar el número de estaciones de monitoreo a fin de obtener más información que permita realizar modelaciones de la concentración anual de MP en LM. Por ejemplo, son pocas las estaciones que registran datos de $\mathrm{PM}_{2.5}$ (Vu et al., 2019). Con ello, se podrían obtener resultados más precisos para la aplicación de políticas públicas, aunque ello no necesariamente conllevaría a un cambio significativo en los costos anuales encontrados en este estudio y/o identificar una tendencia clara de su evolución. Si bien el $\mathrm{PM}_{2.5}$ y $\mathrm{PM}_{10}$ son importantes indicadores de la contaminación del aire, no se debe descartar los impactos ocasionados por ozono, dióxido de azufre el óxido de nitrógeno, lo cuales también contribuirían en menor medida a impactos de mortalidad a la población expuesta.

\section{Literatura citada}

AHRQ [Agency for Healthcare Research and Quality] Medical Expenditure Panel Survey. 2012. Total Expenses and Percent Distribution for Selected Conditions by type of Service: United States. Disponible en: https://meps.ahrq.gov/mepsweb/data_\%20 stats/tables_compendia_hh_interactive. jsp?_SERVICE=MEPSS ocket0\&_\%20 
$\mathrm{P} R \mathrm{O} G \mathrm{R} A \mathrm{M}=\mathrm{M}$ E P S P G $\mathrm{M}$. TC.SAS\&File $=$ HCFY2012\& Table $=H \% 20$ CFY2012_CNDXP_C\&_Debug

Arık, A.; Dodd, E.; Streftaris, G. 2020. Cancer morbidity trends and regional differences in England-A Bayesian analysis. PLOS ONE, 15(5): e0232844.

BCRP [Banco central de reserva del Perú]. 2020. Tipo de cambio (S/ por US\$)Bancario (promedio del periodo) - Compra. Disponible en: https://estadisticas.bcrp. gob.pe/estadisticas/series/anuales/tipo-decambio-sol-usd

Bell, M. L.; Cifuentes, L. A.; Davis, D. L.; Cushing, E.; Telles, A. G.; Gouveia, N. 2011. Environmental health indicators and a case study of air pollution in Latin American cities. Environmental research, 111(1): 57-66.

Cameron, T. A. 2010. Euthanizing the value of a statistical life. Review of Environmental Economics and Policy, 4(2): 161-178.

Chen, J.; Hoek, G. 2020. Long-term exposure to PM and all-cause and causespecific mortality: A systematic review and meta-analysis. Environment international, 143: 105974.

Cohen, A. J.; Brauer, M.; Burnett, R.; Anderson, H. R.; Frostad, J.; Estep, K.; Feigin, V. 2017. Estimates and 25-year trends of the global burden of disease attributable to ambient air pollution: an analysis of data from the Global Burden of Diseases Study 2015. The Lancet, 389(10082): 1907-1918.

Espinoza, J. 2018. Evaluación espacial y temporal del material particulado PM10 y PM2,5 en Lima Metropolitana para el periodo 2015-2017. Tesis de Pregrado en Ingeniería Ambiental. Universidad Nacional Agraria La Molina, Lima. Perú.

Evans, J.; van Donkelaar, A.; Martin, R. V.; Burnett, R.; Rainham, D. G.; Birkett, N. J.; Krewski,D.2013. Estimates of globalmortality attributable to particulate air pollution using satellite imagery. Environmental research, 120: 33-42.

Ferlay, J.; Shin, H.-R.; Bray, F.; Forman, D.; Mathers, C.; Parkin, D. M. 2010. Estimates of worldwide burden of cancer in 2008:
GLOBOCAN 2008. International Journal of Cancer, 127(12).

Fotourehchi, Z. 2016. Health effects of air pollution: An empirical analysis for developing countries. Atmospheric Pollution Research, 7(1): 201-206.

Globocan [Global Cancer Observatory]. 2020. Cancer stories. Disponible en: https://infogram.com/globocan-20201h9j6qg7xdp8v4g?live

Hadei, M.; Shahsavani, A.; Krzyzanowski, M.; Querol, X.; Stafoggia, M.; Nazari, S. S. H., Khosravi, A. 2020. Burden of mortality attributed to PM2. 5 exposures in cities of Iran; contribution of short-term pollution peaks. Atmospheric Environment, 224: 117365.

Han, F.; Lu, X.; Xiao, C.; Chang, M.; Huang, K. 2019. Estimation of Health Effects and Economic Losses from Ambient Air Pollution in Undeveloped Areas: Evidence from Guangxi, China. International journal of environmental research and public health, 16(15): 2707.

IARC [International agency for research on Cancer]. 2014. Diesel and Gasoline Engine Exhausts and Some Nitroarenes. IARC Monographs on the Evaluation of Carcinogenic Risks to Humans Volume 105. Disponible en: https://publications.iarc.fr/129 Ilizarbe, G. M., Valdiviezo, G.; Rojas, J. P.; Cabello, R. J.; Ugarte, C. A. 2020. Chemical Characteristics and Identification of PM10 Sources in Two Lima Districts, Peru. DYNA, 87(215): 57-65.

INEI [Instituto nacional de estadísticas e informática]. 2017a. Población a nivel distrital. Población total al 30 de junio del 2017 , por grupos quinquenales de edad, según departamento, provincia y distrito del año 2015. Disponible en: https://www.inei.gob. pe/estadisticas/indice-tematico/population/

INEI [Instituto nacional de estadísticas e informática]. 2017b. Boletín de Análisis Demográfico $\mathrm{N}^{\circ} 38$. Perú: Estimaciones $\mathrm{y}$ Proyecciones de Población, 1950-2050. Disponible en: https://www.inei.gob.pe/ media/MenuRecursivo/publicaciones_ digitales/Est/Lib1665/libro.pdf 
INEI [Instituto nacional de estadísticas e informática]. 2020. Producto bruto interno por departamentos (cifras preliminares). Disponible en: https://www.inei.gob.pe/ estadisticas/indice-tematico/producto-brutointerno-por-departamentos-9089/

INEN [Instituto Nacional de Enfermedades Neoplásicas]. 2011. Registro de Cáncer de Lima Metropolitana. Incidencia y Mortalidad 2004 - 2005, Volumen 4. Disponible en: http://www.inen.sld.pe/portal/documentos/ pdf/banners_2014/Febrero/25022014_Libro_ RCLM_CD.pdf

INEN [Instituto Nacional de Enfermedades Neoplásicas]. 2016. Registro de Cáncer de Lima Metropolitana. Incidencia y Mortalidad 2010 - 2012, Volumen 5. Disponible en: http://www.inen.sld.pe/ portal/documentos/pdf/banners_2014/2016/ Registro\%20de\%20C\%C3\%A1ncer\%20 Lima\%20Metropolitana\%202010\%20-\%20 2012_02092016.pdf

Jelić, D.; Bencetić Klaić, Z. 2010. Air quality in Rijeka, Croatia. Geofizika, 27(2): 147-167.

Kroeger, T. 2004. Estimación del Costo - Beneficio de medidas para el saneamiento atmosférico. Documento elaborado para el CONAM.

Krzyżanowski, M.; Kuna-Dibbert, B.; \& Schneider, J. (Eds.). 2005. Health effects of transport-related air pollution. WHO Regional Office Europe.

Landrigan, P. J.; Fuller, R.; Acosta, N. J.; Adeyi, O.; Arnold, R.; Baldé, A. B.; Chiles, T. 2018. The Lancet Commission on pollution and health. The lancet, 391(10119): 462-512. Larsen, B.; E. Strukova. 2006. Peru: Cost of Environmental Damage: A SocioEconomic and Environmental Health Risk Assessment. Background Report for Country Environmental Assessment, World Bank, Washington, D.C. October, 2005.

Liangliang, C.; Jingwen, Z.; Xiumiao, P.; Shiman, R.; Zhang, Y. 2020. Analyses of air pollution control measures and co-benefits in the heavily air-polluted Jinan city of China,
2013-2017. Scientific Reports (Nature Publisher Group), 10(1).

Liao, Q.; Jin, W.; Tao, Y.; Qu, J.; Li, Y.; \& Niu, Y. 2020. Health and Economic Loss Assessment of PM2. 5 Pollution during 20152017 in Gansu Province, China. International Journal of Environmental Research and Public Health, 17(9):3253.

Luengo-Fernandez, R.; Leal, J.; Gray, A.; et al. 2013. Economic burden of cancer across the European Union: a population-based cost analysis. Lancet Oncol. 14(12):1165-1174.

Mannucci, P. M., \& Franchini, M. 2017. Health effects of ambient air pollution in developing countries. International journal of environmental research and public health, 14(9), 1048.

Mardones, C., \& Riquelme, M. 2018. Estimation of the value of statistical life in Chile and extrapolation to other Latin American countries. Latin American Research Review, 53(4): 815-830.

Meisner, C., Gjorgjev, D., \& Tozija, F. 2015. Estimating health impacts and economic costs of air pollution in the Republic of Macedonia. South Eastern European Journal of Public Health (SEEJPH).

MINAM [Ministerio de ambiente]. 2017. D.S. $N^{\circ}$ 003-2017-MINAM. Miércoles 7 de junio de 2017. Disponible en: http:// www.minam.gob.pe/disposiciones/decretosupremo-n-003-2017-minam/

MINAM [Ministerio de ambiente]. 2019. Avance: Plan de Acción para el Mejoramiento de la Calidad del Aire de Lima-Callao. Diagnóstico de la Gestión de la Calidad Ambiental del Aire de Lima-Callao. Comisión multisectorial para la gestión de la iniciativa del aire limpio para lima y Callao. Disponible en: https://sinia.minam.gob.pe/documentos/ diagnostico-gestion-calidad-ambiental-airelima-callao

MINSA [Ministerio de salud]. 2012. II Estudio de Saturación Lima Metropolitana y Callao en el año 2011: vigilancia confiable para la protección de la salud de las personas y de su entorno. Elaborado por DIGESA. 
Dirección General de Sanidad Ambiental. Perú. Disponible en: https://sinia.minam. gob.pe/documentos/estudio-saturacion-limametropolitana-callao-ano-2011

MINSA [Ministerio de salud]. 2018. Ministerio de Salud cuenta este año con presupuesto de 733 millones para desarrollar acciones orientadas a combatir el cáncer. Disponible en: https://www.gob.pe/ institucion/minsa/noticias/12842-ministeriode-salud-cuenta-este-ano-con-presupuestode-733-millones-para-desarrollar-accionesorientadas-a-combatir-el-cancer

OECD [Organización para la Cooperación y el Desarrollo Económicos]. 2014. The cost of air pollution: Health impacts of road transport. OECD Publishing. Disponible en: https://read.oecd-ilibrary.org/environment/ the-cost-of-air-pollution_9789264210448en\#page1

Pacsi, S. A. 2016. Análisis temporal y espacial de la calidad del aire determinado por material particulado PM10 y PM2,5 en Lima Metropolitana. Anales Científicos, 77(2), 273-283.

PAHO [Pan American Health Organization]. 2012. Cáncer de Pulmón en las Américas. Disponible en: https://www. paho.org/hq/dmdocuments/2014/OPS-NotaInformativa-Epi-Cancer-Pulmon-2014.pdf

PAHO[PanAmericanHealthOrganization]. 2020. Air quality is deteriorating in many of the world's cities. Pan American Health Organization. Disponible en: https://www. paho.org/hq/index.php?option=com_conten t\&view $=$ article\&id=9558:2014-air-qualityis-deteriorating-in-many-of-the-worldscities\&Itemid=1926\&lang=en

Peng, R. D.; Dominici, F. 2008. Statistical methods for environmental epidemiology with R. R: a case study in air pollution and health.

Quan, M.; Shiqiu, Z. 2015. Assessment of the Trend of Heavy PM2. 5 Pollution Days and Economic Loss of Health Effects during 2001-2013. Acta Scientiarum Naturalium Universitatis Pekinensis, 51(4): 694-706.

Ramos, W.; Guerrero, N.; Medina, J.;
Guerrero, P. 2020. “Análisis de la situación del cáncer en el Perú, 2018”. Lima Ministerio de Salud: Centro Nacional de Epidemiología, Prevención y Control de Enfermedades.

Sánchez, G. S. 2017. Combined application of cost-benefit and multi-criteria analysis for decision support in air quality management policy: a case study in Lima and El Callao, Peru (Doctoral dissertation, Universidad del País Vasco-Euskal Herriko Unibertsitatea).

Seminario, L. 2017. Estimación del Costo Social por Fallecimiento Prematuro. Dirección General de Inversión Pública del Ministerio de Economía y Finanzas. Disponible en: https:// www.mef.gob.pe/contenidos/inv_publica/ docs/parametros_evaluacion_social/Valor_ Estadistico_Vida.pdf

Silva, J.; Rojas, J.; Norabuena, M.; Molina, C.; Toro, R. A.; Leiva-Guzmán, M. A. 2017. Particulate matter levels in a South American megacity: the metropolitan area of LimaCallao, Peru. Environmental monitoring and assessment, 189(12): 635.

Silva, J. S.; Rojas, J. P.; Norabuena, M.; Seguel, R. J. 2018. Ozone and volatile organic compounds in the metropolitan area of LimaCallao, Peru. Air Quality, Atmosphere and Health, 11(8): 993-1008.

Tapia, V.; Carbajal, L.; Vásquez, V.; Espinoza, R.; Vásquez-Velásquez, C.; Steenland, K.; Gonzales, G. F. 2018. Reordenamiento vehicular y contaminación ambiental por material particulado (2, 5 y 10), dióxido de azufre y dióxido de nitrógeno en Lima Metropolitana, Perú. Revista Peruana de Medicina Experimental y Salud Pública, 35: 190-197.

Vu, B. N.; Sánchez, O.; Bi, J.; Xiao, Q.; Hansel, N. N.; Checkley, W.; Liu, Y. 2019. Developing an advanced PM2. 5 exposure model in Lima, Peru. Remote sensing, 11(6): 641.

Wang, H.; Naghavi, M.; Allen, C.; Barber, R. M.; Bhutta, Z. A.; Carter, A.; Coggeshall, M. 2016. Global, regional, and national life expectancy, all-cause mortality, and causespecific mortality for 249 causes of death, 1980-2015: a systematic analysis for the 
Global Burden of Disease Study 2015. The lancet, 388(10053): 1459-1544.

WB \& IHME. 2016. The cost of air pollution: Strengthening the economic case for action. World Bank, \& Institute for Health Metrics and Evaluation. Disponible en: https://lib.icimod.org/record/32239

WB [World Bank]. 2013. Cost Assessment of Environmental Degradation, Institutional Review, and Public Environmental Expenditure Review Country Environmental Analysis Kosovo.http://documents1.worldbank.org/curated/en/282361468047686579/ pdf/750290ESW0P1310LIC00Kosovo0CEA0Rprt.pdf

WB [World Bank]. 2020. Tasa de crecimiento del Producto Bruto Interno de Perú 2001-2007. Disponible en: https://data. worldbank.org/indicator/NY.GDP.MKTP. KD.ZG?locations $=\mathrm{PE}$

Wei, G. R.; Shi, X. M. 2018. Evaluation the Extent of Health Damage Caused by PM2.5 Particulate in Xi'an City. Huan jing ke xue= Huanjing kexue, 39(7): 3014-3021.

WHO [World Health Organization]. 2004. Outdoor air pollution: assessing the environmental burden of disease at national and local levels. Disponible en: https://www. who.int/quantifying_ehimpacts/publications/ ebd5/en/

WHO [World Health Organization]. 2006. Air quality guidelines: global update 2005: particulate matter, ozone, nitrogen dioxide, and sulfur dioxide. World Health Organization. Disponible en: https://www. euro.who.int/en/health-topics/environmentand-health/air-quality/publications/pre2009/ air-quality-guidelines.-global-update-2005.particulate-matter,-ozone,-nitrogen-dioxideand-sulfur-dioxide

WHO [World Health Organization]. 2020. Ambient (outdoor) air quality database, by country and city. Disponible en: https://www. who.int/airpollution/data/cities/en/

Yaduma, N., Kortelainen, M., \& Wossink, A. 2013. Estimating mortality and economic costs of particulate air pollution in developing countries: The case of Nigeria. Environmental and Resource Economics, 54(3): 361-387.

Yu, G.; Wang, F.; Hu, J.; Liao, Y.; Liu, X. 2019. Value assessment of health losses caused by PM2.5 in Changsha City, China. International journal of environmental research and public health, 16(11): 2063. 